\title{
Dutasteride and Prostate Cancer Risk: Does Family History of Prostate and/or Breast Cancers Influence the Number Needed to Treat? Results from REDUCE
}

\author{
Jean-Alfred Thomas II ${ }^{1,2}$, Leah Gerber ${ }^{1,2}$, Robert J. Hamilton ${ }^{3}$, Adriana C. Vidal ${ }^{2,4}$, Daniel M. Moreira ${ }^{5}$, \\ Gerald L. Andriole ${ }^{6}$, Stephen J. Freedland ${ }^{1,2,7, *}$ \\ ${ }^{1}$ Surgery Section, Durham VA Medical Center, Durham, NC \\ ${ }^{2}$ Duke Prostate Center, Division of Urological Surgery, Department of Surgery, Duke, University School of Medicine, Durham, NC \\ ${ }^{3}$ Division of Urology, Department of Surgery, Memorial Sloan Kettering Cancer Institute \\ ${ }^{4}$ Department of Obstetrics and Gynecology, Duke University School of Medicine, Durham, NC \\ ${ }^{5}$ The Arthur Smith Institute for Urology, North Shore Long Island Jewish Health System, New Hyde Park, NY \\ ${ }^{6}$ Washington University School of Medicine in St. Louis, St. Louis, Missouri \\ ${ }^{7}$ Department of Pathology, Duke University School of Medicine, Durham, NC \\ *Corresponding author: Steve.freedland@duke.edu
}

Received June 16, 2014; Revised September 16, 2014; Accepted November 26, 2014

\begin{abstract}
Purpose: In REDUCE, dutasteride was associated with a $5 \%$ absolute reduction in the risk of biopsydetected prostate cancer (PCa). Material and methods: We tested the influence of family history on the association between dutasteride and PCa diagnosis and calculated the number needed to treat (NNT) with dutasteride to avoid one PCa diagnosis. The REDUCE trial tested dutasteride $0.5 \mathrm{mg}$ /day for PCa risk reduction in men aged 50-75 with a serum PSA of $2.5-10.0 \mathrm{ng} / \mathrm{mL}$ and a negative biopsy. Among men who underwent $\geq 1$ on-study biopsy with complete data $(\mathrm{n}=6,415 ; 78.1 \%)$, the association between dutasteride and PCa risk as a function of PCa and/or breast cancer (BCa) family history was examined using multivariable logistic regression. Absolute risk reduction (ARR) and NNT were calculated. Results: On multivariate analysis, dutasteride was significantly associated with lower PCa risk in men without family history ( $25 \%$ lower; $\mathrm{p}<0.001)$, PCa family history only (37\% lower; $\mathrm{p}=0.009$ ), or BCa family history only (38\% lower; $\mathrm{p}=0.04$ ). While dutasteride lowered PCa risk in men with both PCa and BCa family history by $15 \%$, this was not significant $(\mathrm{p}=0.69)$, though the number of men was small $(\mathrm{n}=115)$. ARRs were 6-9\% for men with a PCa and/or BCa family history vs. 5\% in men with no family history which translated into NNTs of 11-16 in men with PCa and/or BCa family history vs. 21 for men without family history. Conclusion: Using dutasteride as a model of chemoprevention, therapies targeting individuals with specific family histories may improve the risk-benefit profile. However, future studies are warranted to confirm our findings.
\end{abstract}

Keywords: prostate cancer, family history, dutasteride

Cite This Article: Jean-Alfred Thomas II, Leah Gerber, Robert J. Hamilton, Adriana C. Vidal, Daniel M. Moreira, Gerald L. Andriole, and Stephen J. Freedland, "Dutasteride and Prostate Cancer Risk: Does Family History of Prostate and/or Breast Cancers Influence the Number Needed to Treat? Results from REDUCE." American Journal of Cancer Prevention, vol. 2, no. 2 (2014): 31-36. doi: 10.12691/ajcp-2-2-3.

\section{Introduction}

Given its prolonged natural history, prostate cancer (PCa) is an ideal target for chemoprevention strategies aimed to reduce disease incidence. Many of the proposed candidates (e.g. selenium, vitamin E) have fallen short of the goal of lowering PCa risk, whereas 5- $\alpha$ reductase inhibitors (5-ARIs) such as finasteride and dutasteride have been shown to be associated with lower PCa risk in randomized trials [1,2,3]. However, some feel institution of widespread use of chemopreventitive agents may not be a cost- effective means of impacting PCa incidence [4]. Furthermore, many are concerned with relatively unfavorable side-effect profile (e.g. decreased libido, erectile dysfunction, risk of high-grade PCa). Indeed, the FDA ultimately ruled that the use of 5-ARIs did not possess a reasonable risk-benefit profile for use in healthy men and these agents are not approved as such and the makers of finasteride and dutasteride are not seeking approval for these indications nor advocate their use for this purpose [5]. Thus, these interventions have not been broadly adopted in the general population [6]. Nonetheless, some investigators propose that a more selective use of these agents focusing on individuals who may be deemed as "high risk" for future PCa diagnosis would serve to improve the risk-benefit profile [7]. One group in particular who may stand to benefit from this is individuals with a positive PCa family history. Indeed, 
men with at least one affected first-degree relative with $\mathrm{PCa}$ are at more than 2-fold increased risk for future PCa diagnosis versus those with no family history [8]. Similarly thought to be at increased PCa risk are men with breast cancer (BCa) family history alone or in combination with PCa family history and as such may likewise benefit [9]. Especially given that the latter group may have a higher proportion of individuals with BRCA1 and -2 , mutations known to be associated with elevated risk of PCa [10].

How well certain chemopreventive strategies (i.e. 5ARIs) work to reduce PCa risk as a function of family history has been examined, though only considering presence or absence of general PCa family history [11]. Using data from REDUCE, a 4- year, placebo-controlled randomized clinical trial of dutasteride for the chemoprevention of PCa, we previously showed that certain groups based upon family history are at increased risk of PCa [12]. Specifically, men with either a PCa family history or a family history of both PCa and BCa had significantly increased PCa risk. On the contrary, those with a BCa family history alone had similar PCa risk as men with no family history. Thus, men could be categorized into a lower-risk group (no family history of family history of BCa alone) or a higher-risk group (PCa family history or both PCa and BCa family history). Though we found no significant interaction between dutasteride and family history, the statistical bar for finding a significant interaction is quite high, especially with only modest numbers of men in certain family history subgroups. As such, herein we performed a more thorough analysis of how family history may influence the association between dutasteride use and PCa risk. Finally, and of most clinical relevance, we determined the number of men that needed to be treated (NNT) with dutasteride to avoid one diagnosis of $\mathrm{PCa}$, which was the primary outcome of this study [12].

Of note, the goal of this study was to examine the impact of differing baseline PCa risk on the impact of an agent shown to reduce PCa risk. As only finasteride and dutasteride have been shown in phase III studies to reduce PCa risk, we choose to study 5ARIs. However, we must reiterate that the use of dutasteride or finasteride has not been approved for the purposes of PCa prevention in the United States or Europe [13,14]. Whether these are the best agents to use in clinical practice remains the decision of the treating physician and patient. As such, we used dutasteride as an example of chemoprevention rather than endorsing or condoning its use for chemoprevention.

\section{Study Subjects and Methods}

\subsection{Study Population}

The design of the REDUCE study has been reported [3]. Eligible men were aged 50-75 years, with a serum PSA of $2.5-10 \mathrm{ng} / \mathrm{mL}$ if aged $50-60$ years, or $3-10 \mathrm{ng} / \mathrm{mL}$ if $>60$ years, and a single, negative prostate biopsy (6-12 cores) within 6 months prior to enrollment. This resulted in a population considered to be at increased risk of prostate cancer.

\subsection{Study Design}

REDUCE was a 4-year, multicenter, double-blind, placebo-controlled study. Eligible subjects were randomized to dutasteride $0.5 \mathrm{mg} /$ day or placebo. Visits occurred every 6 months. Total serum PSA (Beckman Coulter Inc.) was assessed every 6 months, with doubled PSA values $( \pm 0.1 \mathrm{ng} / \mathrm{mL}$ ) reported to investigators for men receiving dutasteride. Unscheduled PSA measurements were permitted if obtained through the central study laboratory.

Subjects underwent a 10-core transrectal ultrasound (TRUS)-guided biopsy at 2 and 4 years regardless of PSA levels ("protocol-dependent" biopsies); unscheduled biopsies were performed if clinically indicated ("protocol-independent" biopsies). Protocol-independent biopsies obtained during Months 19-24 and 43-48 replaced those scheduled for Years 2 and 4, and were included in the definition of protocol-dependent biopsies.

At baseline, a detailed medical history was obtained including family history of PCa or BCa defined as at least one single first-degree relative affected. Race was selfreported. Digital rectal examination (DRE) findings and TRUS prostate volume were reported from the pre-study biopsy.

\subsection{Statistical Analyses}

Among the 8,122 men in the efficacy population, 6,729 (82.8\%) had at least one on-study biopsy. There were no significant differences in the distribution of self-reported PCa or BCa family history between men who did and did not undergo biopsy (20.9\% vs. $\left.22.2 \%, x^{2} \mathrm{p}=0.30\right)$. Men who did not undergo a biopsy were similar aged, had similar baseline PSA, body mass index (BMI), and DRE findings (all $\mathrm{p}>0.05$ ). Men who did not undergo a biopsy were more likely to be black ( 3.9 vs. $1.9 \%$; $<<0.001$ ). Details of the biopsy population have been published [3]. Of the 6,729 men who had at least one on- study biopsy, we excluded men with missing data for pre-study PSA $(n=15)$, BMI $(n=204)$, DRE $(n=7)$, TRUS volume $(n=76)$, or benign prostatic hyperplasia $(\mathrm{BPH} ; n=1)$, resulting in a final population of 6,426 all of whom had family history data.

Study participants were grouped according to PCa or BCa family history as following: no cancer family history, PCa family history only, BCa family history only, or PCa and BCa family history.

The distribution of baseline characteristics by treatment arm was examined using descriptive statistics. A crude rate of PCa incidence was generated from which the absolute risk reductions (ARR) were calculated. From this, we calculated the NNT with dutasteride to avoid one PCa diagnosis (1/ARR), which was the primary outcome of this current analysis. In addition, we tested the association between treatment arm and PCa diagnosis as a function of family history subgroups using multivariable logistic analyses with dutasteride as the predictor and PCa as outcome. Results were adjusted for clinical characteristics associated with PCa risk including age (continuous), race (white, black and other), baseline PSA (log-transformed, continuous), prostate volume (log- transformed, continuous), DRE findings (abnormal vs. normal), BMI (logtransformed, continuous), and geographic region. Finally, we tested whether family history modified the association between treatment arm and PCa incidence by formal 
interaction testing by including a categorized crossproduct term (treatment arm X family history category; 3 degrees of freedom) along with both primary variables in the logistic regression model. All analyses were conducted using Stata 11.1 (STATA corp.; College Station, TX) with a p-value $\leq 0.05$ indicative of significance.

\section{Results}

\subsection{Study Population and Baseline Characteristics}

Overall, the treatment arms were well balanced in terms of baseline characteristics (Table 1). Most men had no PCa or BCa family history ( $n=5,008 ; 77.9 \%$; Table 2). There were 717 (11.1\%) men with only PCa family history, 581 (9\%) with only BCa family history, and 121 (1.9\%) with both. Men with a PCa and/or BCa family history were younger, had smaller prostates, were more likely to have an abnormal DRE, and had lower baseline PSA values versus men with no family history (all p values $<0.05$ ), though differences were slight.
Table 1. Baseline Characteristics of Study Population by Treatment Arm

Number of patients

Median Age at study enrollment

(years)

Ethnicity, n(\%)

$\begin{array}{lcc}\text { White } & 2,897(92) & 2,996(92) \\ \text { Black } & 59(2) & 60(2) \\ \text { Other } & 201(6) & 213(6)\end{array}$

Geographic Region, n (\%)

North America 755 (24) 799 (24)

South America 419 (13) 415 (13)

Europe $\quad 1,832(58) \quad 1,896(58)$

Australia/New Zealand 60 (2) 62 (2)

Other 91 (3) 97 (3)

Body Mass Index $\left(\mathrm{kg} / \mathrm{m}^{2}\right) \quad 26.9(24.8-\quad 26.8(24.8-$

Median PSA, ng/ml (IQR) $\quad 5.7$ (4.4-7.3) 5.7 (4.4-7.3)

Median TRUS, cc (IQR) $\quad 43.4$ (33.1- 43.4 (33.1-

$117(4) \quad 124(4)$

Table 2. Baseline Characteristics of Study Population by Family History

\begin{tabular}{|c|c|c|c|c|c|}
\hline \multirow[b]{3}{*}{ Number of patients, n (\%) } & \multicolumn{4}{|c|}{ Family History of Prostate and/or Breast Cancer } & \\
\hline & No Family History & Prostate cancer & Breast Cancer & Prostate \& Breast Cancer & \\
\hline & 5,008 (77.9) & $717(11.2)$ & $580(9.0)$ & $121(1.9)$ & p-value \\
\hline Median Age at study enrollment (years) & $63(59-68)$ & $61(56-65)$ & $62(58-66)$ & $61(57-66)$ & $<0.001^{\dagger}$ \\
\hline \multicolumn{6}{|l|}{ Race, n (\%) } \\
\hline White & $4,574(91)$ & $660(92)$ & $540(93)$ & $119(98)$ & $0.027 *$ \\
\hline Black & $89(2)$ & $17(2)$ & $13(2)$ & $0(0)$ & \\
\hline Other & $345(7)$ & $40(6)$ & $27(5)$ & $2(2)$ & \\
\hline Geographic Region, n (\%) North America & $1,006(20)$ & $289(40)$ & $190(33)$ & $69(57)$ & $<0.001^{*}$ \\
\hline South America & $681(14)$ & $67(12)$ & $82(12)$ & $4(3)$ & \\
\hline Europe & $3,070(61)$ & $325(45)$ & $289(50)$ & $44(36)$ & \\
\hline Australia/New Zealand & $83(2)$ & $11(2)$ & $25(4)$ & $3(3)$ & \\
\hline Other & $168(3)$ & $10(1)$ & $9(1)$ & $1(1)$ & \\
\hline Body Mass Index (kg/m²) & $26.8(24.8-29.2)$ & $27.1(25.1-29.6)$ & $26.9(24.8-29.4)$ & $26.7(24.6-29.2)$ & $0.26^{\S}$ \\
\hline Median PSA, ng/ml (IQR) & $5.8(4.5-7.4)$ & $5.4(4.1-7.0)$ & $5.5(4.3-7.1)$ & $5.4(4.3-7.1)$ & $<0.001^{\S}$ \\
\hline Median TRUS, cc (IQR) & $43.8(33.2-56.9)$ & $41.5(31.5-55.7)$ & $43.4(33.8-55.1)$ & $41.8(32.9-52.3)$ & $0.009^{\S}$ \\
\hline Abnormal Digital Rectal Exam, n (\%) & $166(3)$ & $33(5)$ & $28(5)$ & $14(12)$ & $<0.001^{*}$ \\
\hline Assigned to Dutasteride arm, n (\%) & & & & & $0.38^{*}$ \\
\hline No & $2,576(51)$ & 354 (49) & $280(48)$ & $59(49)$ & \\
\hline Yes & $2,432(49)$ & $363(51)$ & $300(52)$ & $62(51)$ & \\
\hline
\end{tabular}

PSA prostate-specific antigen; TRUS transrectal ultrasound

P-value calculated using ${ }^{\dagger}$ ANOVA, *Kruskal-Wallis, or ${ }^{\S_{C}}$ Chi-squared test

†Multivariable logistic regression analyses adjusted for age, race, PSA, BMI, TRUS volume, geographic region, DRE findings, and treatment arm.

\subsection{Risk Reduction, Dutasteride, and Family History}

Among men with no PCa or BCa family history who made up the vast majority, dutasteride reduced PCa diagnosis by 25\% (Table 3). Among men with either a PCa or BCa family history alone, dutasteride use was associated with a $38 \%$ and $35 \%$ reduced risk of $\mathrm{PCa}$, respectively. Among men with a family history of both PCa and BCa family history, dutasteride was associated with a non-significant $19 \%$ PCa risk reduction, though the number of men with a family history of both cancers was small $(\mathrm{n}=121)$.

Table 3. Prostate Cancer Risk Reduction by Dutasteride As a Function of Family History of Prostate and/or Breast Cancers ${ }^{\dagger}$

\begin{tabular}{lcc}
\hline & Risk Reduction (1-OR) & p-value \\
No family history of cancer & $25 \%(14 \%-35 \%)$ & $<0.001$ \\
Prostate cancer only & $38 \%(12 \%-56 \%)$ & 0.007 \\
Breast cancer only & $35 \%(2 \%-57 \%)$ & 0.041 \\
Prostate and Breast cancer & $19 \%(-8 \%-65 \%)$ & 0.61 \\
\hline
\end{tabular}


When examining PCa incidence across family history groups, dutasteride was associated with 5\% ARR in men with no family history of cancer and a 6\% AAR in men with a BCa family history only (Figure 1). This translated into a NNT to avoid one PCa diagnosis of 16 and 21, respectively. However, in subgroups that we have previously shown within REDUCE are at increased risk of
PCa diagnosis (i.e. those with PCa alone or PCa and BCa family history), dutasteride was associated with a 9\% ARR. This translated into a NNT of 11-12. When formal statistical tests for interaction were examined, family history did not appear to significantly modify the association between dutasteride and prostate cancer (pinteraction $>0.30$ ).

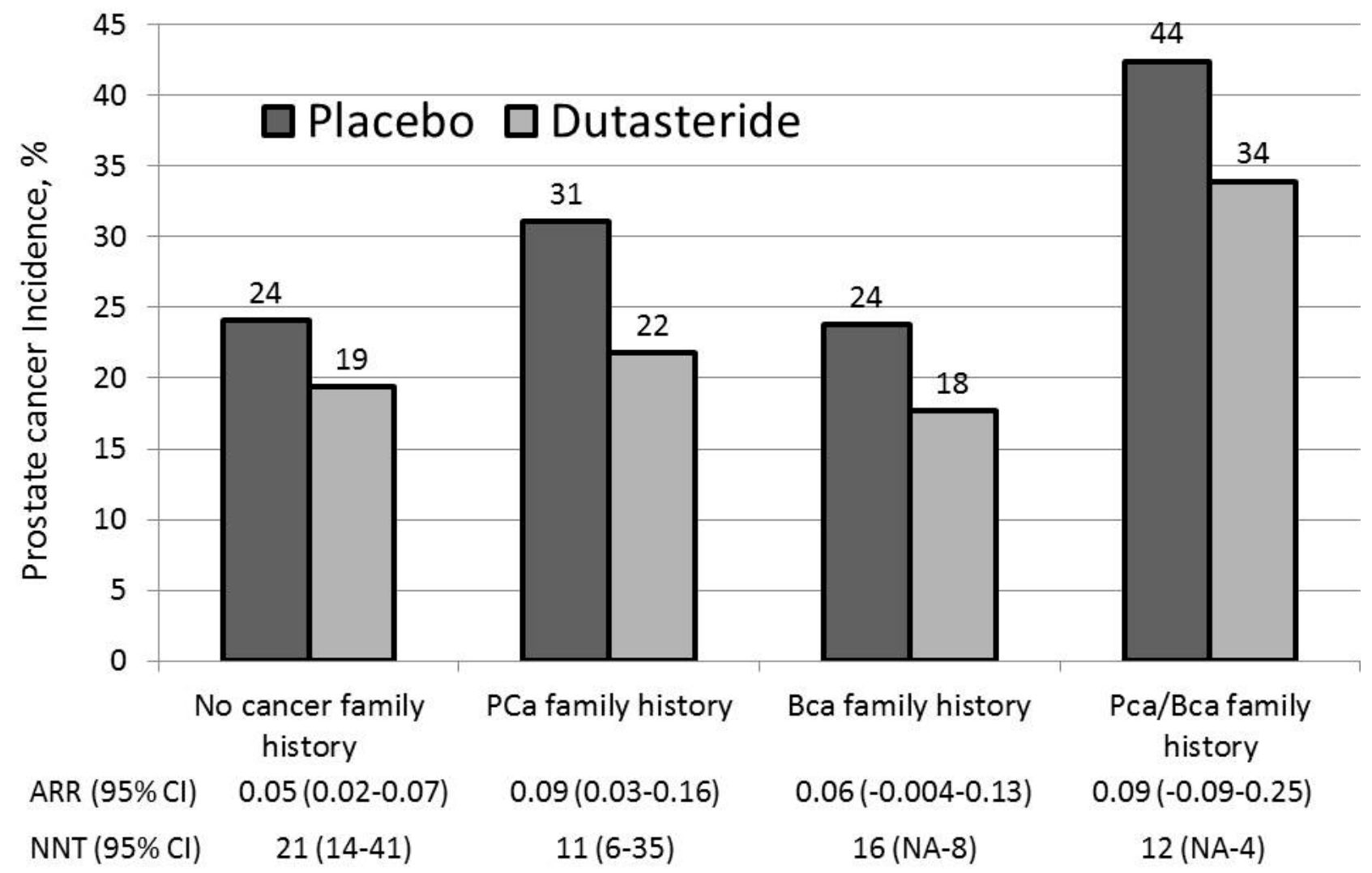

ARR: Absolute Risk Reduction

NNT: number needed to treat

Figure 1. Prostate cancer incidence across family history groups. Dutasteride was associated with $5 \%$ ARR in men with no family history of cancer, $6 \%$ AAR in men with a BCa family history only. In men with family history of PCa alone or PCa and BCa family history, dutasteride was associated with a 9\% ARR. NNT to avoid one PCa diagnosis was 16 and 21, respectively

\section{Discussion}

Concerns over cost-effectiveness and side effects are important considerations in the use of chemoprevention in the general, otherwise healthy population. As such, dutasteride as an example of a chemopreventative agent is no different. Though its use has been associated with a significant reduction in $\mathrm{PCa}$ risk in a randomized controlled trial, due to concerns about side effects, including the possibility of increased risk of high-grade disease, its use of PCa prevention is not approved by the FDA [15]. However, with any intervention, the riskbenefit equation may be different in different subgroups. As such, the goal of this study was to use the example of dutasteride as an agent shown to reduce PCa risk and identify the effect of differing baseline PCa risk using family history $[11,16]$ on the NNT of a chemopreventative agent.

Data from the original REDUCE study suggest dutasteride lowers the relative risk risk of PCa diagnosis by $~ 23 \%$ (3). The authors of the original REDUCE trial reported no significant difference in dutasteride's ability to reduce disease incidence by presence or absence of PCa family history. In a subsequent analysis, we found no significant interaction between dutasteride and family history [12]. However, when subgroup sizes are relatively small, a large difference between outcomes in each subgroup is needed to drive the statistical test of interaction to significance. As such, in the current report we performed a more detailed examination of the association between dutasteride use and overall PCa risk across family history groups using the REDUCE trial. Of note, in our prior report, we found that men with PCa family history alone or PCa and BCa family history were at increased risk of PCa diagnosis [12]. In the current study, dutasteride was associated with lower risk of PCa diagnosis across all family history subgroups, though this was not statistically significant for men with both PCa and BCa family history.

Given reservations with respect to the costeffectiveness and risk-benefit profile of dutasteride -or any chemoprevention modality-, some authors have previously suggested that the focus of such strategies should be directed to high-risk men such as those with family history of disease [16]. We found that 21 men 
without a family history of PCa or BCa would have to undergo dutasteride chemoprevention to prevent one cancer diagnosis, whereas only 11, 16, and 12 men with PCa, BCa, or PCa and BCa family histories, respectively, would similarly have to be treated to avoid a single diagnosis over a 4-year period, of course being mindful of the absence of any statistical significance as it relates to men with both PCa and BCa family history- a potential consequence of small numbers in this subgroup. By comparison, studies of statin use and the risk of myocardial infarction or stroke have observed that the number needed to treat to prevent one myocardial infarction or stroke over 5 years among patients with family history or hyperlipidemia is 12-20 [17]. As such, the NNT with an agent that reduced PCa risk by $\sim 25 \%$ (i.e. a 5-ARI) to avoid one PCa diagnosis is comparable to the NNT with a statin to prevent a myocardial infarction or stroke over a similar period of time. Moreover, selecting patients at higher risk would not only reduce the total number of patients to be treated with chemoprevention to prevent PCa, but also improve the long-term costeffectiveness of chemoprevention. For example, Reed et al. reported the cost-effectiveness of chemoprevention with finasteride is greatest in men with a positive family history and higher numbers of inherited risk alleles (16).

To assess any chemoprevention agent in its entirety, one must take into consideration not only the NNT but the number needed to harm (NNH) as it pertains to any adverse event, be it high-grade disease or any other side effect. Specifically, dutasteride was linked with increased risk of Gleason 8 or higher in an FDA re-review of the biopsy slides from REDUCE, but not in the original manuscript [5]. As such, given the FDA reviewed data were not available to us and dutasteride was not linked with high-grade disease in the original REDUCE study, we did not examine the NNH related to high-grade disease [18]. Again, the goal of this study was to use dutasteride as an example of chemoprevention rather than provide specific data to support or refute its use.

We recognize several limitations. First, more detailed family history data were unavailable (e.g. second-degree relatives). As family history is a summation of genetic and environmental factors, we cannot fully address to what degree shared environmental factors vs. genetics dictated the observed associations. Thus, it may be possible in the future to define an even higher-risk cohort using not just family history, but genetic data wherein the NNT may be even lower. Ultimately, this is a secondary analysis of randomized trial wherein men were in receipt of biopsy prior to study enrollment. As such, we cannot exclude the possibility that these results may differ from a biopsynaïve population. Third, the number of men with highgrade and advanced disease were few preventing any meaningful analyses, though as noted dutasteride has either no effect (original REDUCE observations) or actually increased the risk of Gleason 8-10 tumors (FDA re-review). Despite these limitations, our study has key strengths. This study is unique in that men underwent biopsies regardless of PSA allowing us to test the association between dutasteride use and PCa diagnosis as a function of family history while minimizing screening biases.

In summary, in a cohort wherein all men underwent prostate needle biopsy regardless of PSA, dutasteride was associated with a reduced risk of PCa across all family history subgroups. However, due to the higher-risk nature of men with either PCa or PCa and BCa family history, the NNT was lower at 11-12 vs. men without a family history of PCa wherein the NNT was 21. Using dutasteride as a model of chemoprevention, therapies targeting individuals at high-risk - in this case those with PCa or PCa and BCa family history - may prove an effective and reasonable way to reduce risk among these high-risk subgroups while minimizing the exposure of lower- risk individuals to unwanted side effects and improving cost-effectiveness.

\section{Disclosure of Potential Conflicts of Interest}

Dr. Andriole received consulting/advisory and lecture fees from GSK. Dr. Freedland received research funding from GSK.

\section{Acknowledgements}

The authors wish to acknowledge the dedication of the patients, investigators, data and safety monitoring committee, steering committee, and GSK in the initiation and conduct of the REDUCE study.

\section{Grant Support}

This study was supported by NIH K24 CA160653-01 grant to Dr. Freedland and support from GSK for data analysis.

\section{References}

[1] Lippman SM, Klein EA, Goodman PJ, Lucia MS, Thompson IM, Ford LG, et al. Effect of selenium and vitamin E on risk of prostate cancer and other cancers: the Selenium and Vitamin E Cancer Prevention Trial (SELECT). JAMA. 2009; 301: 39-51.

[2] Thompson IM, Goodman PJ, Tangen CM, Lucia MS, Miller GJ, Ford LG, et al. The influence of finasteride on the development of prostate cancer. N Engl J Med. 2003; 349: 215-24.

[3] Andriole GL, Bostwick DG, Brawley OW, Gomella LG, Marberger M, Montorsi F, et al. Effect of dutasteride on the risk of prostate cancer. N Engl J Med. 2010; 362: 1192-202.

[4] Svatek RS, Lotan Y. Cost utility of prostate cancer chemoprevention with dutasteride in men with an elevated prostate specific antigen. Cancer Prev Res (Phila). 2011; 4: 277-83.

[5] Theoret MR, Ning YM, Zhang JJ, Justice R, Keegan P, Pazdur R. The risks and benefits of 5alpha- reductase inhibitors for prostatecancer prevention. N Engl J Med. 2011; 365: 97-9.

[6] Hamilton RJ, Kahwati LC, Kinsinger LS. Knowledge and use of finasteride for the prevention of prostate cancer. Cancer Epidemiol Biomarkers Prev. 2010; 19: 2164-71.

[7] Svatek RS, Lee JJ, Roehrborn CG, Lippman SM, Lotan Y. The cost of prostate cancer chemoprevention: a decision analysis model. Cancer Epidemiol Biomarkers Prev. 2006; 15: 1485-9.

[8] Johns LE, Houlston RS. A systematic review and meta-analysis of familial prostate cancer risk. BJU Int. 2003; 91: 789-94.

[9] Cerhan JR, Parker AS, Putnam SD, Chiu BC, Lynch CF, Cohen $\mathrm{MB}$, et al. Family history and prostate cancer risk in a populationbased cohort of Iowa men. Cancer Epidemiol Biomarkers Prev. 1999; 8: 53-60.

[10] Liede A, Karlan BY, Narod SA. Cancer risks for male carriers of germline mutations in BRCA1 or BRCA2: a review of the 
literature. Journal of clinical oncology: official journal of the American Society of Clinical Oncology. 2004; 22: 735-42.

[11] Madersbacher S, Alcaraz A, Emberton M, Hammerer P, Ponholzer A, Schroder FH, et al. The influence of family history on prostate cancer risk: implications for clinical management. BJU international. 2011; 107: 716-21.

[12] Thomas JA, 2nd, Gerber L, Moreira DM, Hamilton RJ, Banez LL, Castro-Santamaria R, et al. Prostate cancer risk in men with prostate and breast cancer family history: results from the REDUCE study (R1). Journal of internal medicine. 2011.

[13] Vollmer RT, Montana GS. Predicting tumor failure in prostate carcinoma after definitive radiation therapy: limitations of models based on prostate-specific antigen, clinical stage, and Gleason score. Clin Cancer Res. 1999; 5: 2476-84.

[14] Freedland SJ, Jalkut M, Dorey F, Sutter ME, Aronson WJ. Race is not an independent predictor of biochemical recurrence after radical prostatectomy in an equal access medical center. Urology. 2000; 56: 87-91.
[15] Wilt TJ, Macdonald R, Hagerty K, Schellhammer P, Tacklind J, Somerfield MR, et al. 5-alpha- Reductase inhibitors for prostate cancer chemoprevention: an updated Cochrane systematic review. BJU international. 2010; 106: 1444-51.

[16] Reed SD, Scales CD, Jr., Stewart SB, Sun J, Moul JW, Schulman $\mathrm{KA}$, et al. Effects of family history and genetic polymorphism on the cost-effectiveness of chemoprevention with finasteride for prostate cancer. J Urol. 2011; 185: 841-7.

[17] Ridker PM, MacFadyen JG, Fonseca FA, Genest J, Gotto AM, Kastelein JJ, et al. Number needed to treat with rosuvastatin to prevent first cardiovascular events and death among men and women with low low-density lipoprotein cholesterol and elevated high-sensitivity C-reactive protein: justification for the use of statins in prevention: an intervention trial evaluating rosuvastatin (JUPITER). Circ Cardiovasc Qual Outcomes. 2009; 2: 616-23.

[18] Theoret MR, Ning YM, Zhang JJ, Justice R, Keegan P, Pazdur R. The risks and benefits of 5alpha- reductase inhibitors for prostatecancer prevention. The New England journal of medicine. 2011; 365: 97-9. 